\title{
Understanding statistics 1
}

\author{
Elliot Abt
}

Department of Dentistry, Illinois Masonic Medical Center, Chicago, Illinois, USA

Understanding the basics of statistics can be quite challenging. One of the problems with statistical analysis is the manner in which it is presented, which is often rather disjointed. This series of articles will attempt to approach statistics by keeping issues in specific categories, to try to facilitate understanding of key concepts. This first article will examine the need for statistical analysis, explore the development of the null hypothesis, and describe what general domains of statistics are used in scientific studies.

\section{When is statistical analysis needed?}

Research can generally be categorised into either quantitative and qualitative studies. Quantitative research may be familiar to clinicians as it represents scientific discovery through measurement. Whether one is examining failure rates with different prosthetic restorations or evaluating changes in pocket depth with periodontal interventions, these studies attempt to quantify outcomes. The investigator is an unbiased observer who uses instruments or questionnaires to gather information, and inferential statistics so that data can then be extrapolated from the samples and applied to the general population.

Qualitative research generally focuses on exploring the experiences of subjects in different situations and environments. Qualitative investigations involve analysis of non-numerical data such as the thoughts, feelings and opinions of those being studied, often through the use of interviews and focus groups. The investigators are immersed in the study and function as the data-gathering instruments. If statistics are presented, they are usually descriptive in nature, such as simple proportions (percentages). Studies examining the expectations of orthodontic patients, why dentists choose to specialise, or why patients are fearful of dental visits, may lend themselves to qualitative investigations.

The role of clinical dental research is to make advances in knowledge that culminate in improved patient care. Clinical studies and their statistical analysis are not of added benefit or very useful in all circumstances, however: when the benefits of a therapeutic intervention are obvious, scientific study is unnecessary. Conversely, research can provide clarity when differences (or associations) between groups are subtle, or uncertain. For example, one might have some difficulty recruiting oral-surgery patients for a clinical trial comparing the use of a local anaesthetic to a placebo. Not only would this trial be unethical, it would also be unnecessary as local anaesthetics provide an obvious therapeutic benefit. The effects of one type of anaesthetic compared with another, though, are less certain, and randomised trials have examined the subtleties of these differences. ${ }^{1}$ Unfortunately, obvious therapeutic benefits that preclude scientific study are rare with medical and dental therapies. Because most interventions are characterised by a moderate to high level of uncertainty, research demands remain high in healthcare.

\section{What is the null hypothesis?}

Fundamental to statistical analysis is the concept of the null hypothesis. Evolving from both inductive and deductive reasoning, the null hypothesis was developed because it is easier to disprove than to prove a hypothesis. For example, if one's hypothesis is that all denture wearers are dissatisfied, how many unhappy denture wearers would have to be identified to prove this? It is arguable that proof would be theoretically impossible, whereas finding just one satisfied denture wearer effectively disproves this hypothesis. Therefore, scientific investigations begin by establishing a null hypothesis, which states there is no difference (or association) between groups, and an alternative hypothesis, which states there is a difference between groups. Investigators set out believing the null hypothesis, until there is strong evidence that it is not true - ie, when the evidence indicates a low probability that the null hypothesis is true. The investigators will either fail to reject, or will reject, the null hypothesis in favour of the stated alternative hypothesis. Thus, scientific advances are accomplished by attempting to disprove the null hypothesis.

\section{What do statistics accomplish?}

Readers of the dental literature will often find articles that attempt to show that one particular therapy is more effective than another, but statistics can also demonstrate when there is a relationship between two or more variables. We will consider three general domains of what statistics can do for scientific investigations:

1. Differences between groups

2. Associations between groups

3. Time-to-event data

\section{Differences between groups}

This most frequently encountered domain examines whether two or more groups are different from each other. Investigations will examine if one treatment is different (better or worse) than another. Changes in cholesterol level, alveolar bone levels or post-operative pain are common outcomes representative of this domain.

\section{Association between groups}

A less common domain seen in scientific studies is association between groups, a domain that is often reported by the news 
media. Examples include associations between red wine consumption and cardiac health, coffee consumption and liver disease, or living near high-tension wires and cancer risk. The relationship between periodontal disease and coronary artery disease is a rather popular topic in dentistry and representative of this domain. One of the major caveats with this domain is that an association between variables does not imply causation. In fact, associations can be rather easy to show. If one looked for a statistical association between periodontal disease and grey hair, one is likely to find it, as both variables are associated with ageing.
Clearly, we would not conclude that either variable caused the other. In this example, age is a confounder, a factor related to both exposure and outcome, which tends to show a causative relationship between variables when, in fact, none exists.

\section{Time-to-event (survival) data}

Time-to-event data, also referred to as survival data, measure the length of time to an event. These events can vary and include number of days in a hospital, survival, time in remission or death. Many cancer studies use statistical tests in this domain. Time to failure of a dental prosthesis is a com- mon dental outcome using survival data. Although this domain also examines differences between groups, it is presented separately as it employs a different set of statistical tests.

The next article in this series will review the different types of data, which generally determine the type of statistical test(s) used in a study.

1. Abdulwahab M, Boynes $S$, Moore $P$, et al. The efficacy of six local anesthetic formulations used for posterior mandibular buccal infiltration anesthesia. J Am Dent Assoc 2009; 140: 1018-1024.

Evidence-Based Dentistry (2010) 11, 60-61. doi:10.1038/sj.ebd.6400728. 